\title{
La enseñanza de la lectoescritura en El Salvador: Perspectivas durante la pandemia de COVID-19
}

\author{
Mario Zetino \\ Licenciado en Letras \\ Investigador \\ Universidad Dr. José Matías Delgado, El Salvador \\ mesermenoz@ujmd.edu.sv \\ ORCID: https://orcid.org/0000-0003-0573-2449
}

Recepción: 03-09-2020 / Aceptación: 07-01-2021

\section{Resumen}

El artículo tiene por objetivo evaluar la enseñanza de la lectoescritura en la Educación Básica y Media en el sistema público de El Salvador durante la pandemia de COVID-19.

La revisión de la literatura resume los primeros análisis sobre el tema, y también recoge estudios que tocan un punto medular, como es la formación docente para la lectoescritura. Se analizan las medidas adoptadas y los medios utilizados para la continuidad del desarrollo de las competencias de lectura y escritura, y de ese modo se hace una revisión de la capacitación docente, los programas de estudio, los libros de texto, las guías de aprendizaje y las teleclases.

Como conclusiones se identifican la urgencia de una formación docente adecuada para una enseñanza de calidad de la lectoescritura, así como elementos funcionales y puntos de mejora en los medios y prácticas educativos utilizados durante este período.

Palabras clave: Lectoescritura, competencias, formación docente, COVID-19.

\begin{abstract}
The objective of this article is to evaluate the teaching of literacy in Basic and Secondary Education in the public system of El Salvador during the COVID-19 pandemic.

The literature review summarizes the first analyses on the subject, and also includes studies that touch on the core issue of teacher training for reading and writing. It analyzes the measures adopted and the means used for the continued development of reading and writing skills, and thus reviews teacher training, curricula, textbooks, learning guides and teleclasses.

As conclusions, the urgency of adequate teacher training for quality literacy teaching is identified, as well as functional elements and points for improvement in the educational means and practices used during this period.
\end{abstract}

Key words: Literacy, competencies, teacher training, COVID-19. 


\section{Introducción}

Las competencias de lectura, escritura y aritmética son competencias básicas de participación y factores de desarrollo dentro de la sociedad actual. Al mismo tiempo, son metas de los Objetivos de Desarrollo Sostenible (ODS) 2030 de la Organización de las Naciones Unidas (UNESCO, 2017); por lo que su desarrollo debe ser una prioridad para todo sistema e institución educativa. La pandemia de COVID-19 ha significado un desafío enorme para la educación a nivel mundial, y como parte de ese desafío se plantea la continuidad del desarrollo de las competencias antes mencionadas.

Este artículo aborda la enseñanza de la lectoescritura en El Salvador en los niveles de Educación Básica y Media en el sistema público en el contexto de la pandemia. Se exponen las medidas tomadas para dicho fin; se evalúan diversas prácticas y materiales, se señalan elementos funcionales y áreas de mejora en estos; también, se formulan recomendaciones para mejorar la enseñanza de dichas competencias. Se presenta un contexto de la enseñanza en El Salvador durante la pandemia. Para esto se siguen los estudios de Arévalo y Hernández (2020), y Picardo, Ábrego y Cuchillac (2020).

\section{Gestión de la educación en el sistema público}

Los estudios califican las acciones del Ministerio de Educación, Ciencia y Tecnología (MINEDUCYT) ${ }^{1}$ durante la pandemia como centralizantes (Arévalo y Hernández, 2020; Picardo, Ábrego y Cuchillac, 2020).
De acuerdo a Picardo, Ábrego y Cuchillac (2020), "[durante la pandemia] el MINEDUCYT centraliza las propuestas didácticas en el sector público. Es decir, disminuye el papel del docente en el planteamiento didáctico pedagógico y curricular” (p. 36). Por su parte, Arévalo y Hernández (2020) afirman que el rol de los docentes fue relegado a "la intermediación de guías [de aprendizaje] entre los estudiantes y el Ministerio" (p. 73).

Profundizando dicho análisis, Arévalo y Hernández (2020) caracterizan los procesos educativos realizados en este período como de corte bancario, mediados por el uso de la internet (p. 74). Al respecto, los investigadores señalan que "el MINEDUCYT se enfocó en la 'continuidad del desarrollo del currículo"' (p. 81): "Al parecer, el desarrollo del currículo se interacciona [sic] como sinónimo de aprendizaje [...], por lo cual el personal docente se enfocó en el desarrollo curricular, sin poder matizar con elementos de contexto para promover un aprendizaje más significativo en el estudiantado" (p. 81).

Las mayores dificultades para una continuidad educativa por medio de internet son: la carencia de recursos económicos y tecnológicos (el equipo y la conectividad adecuados), tanto de los centros escolares como de los docentes y estudiantes; y la baja alfabetización tecnológica, que es mayor en los docentes (Arévalo y Hernández, 2020; Picardo, Ábrego y Cuchillac, 2020). A continuación, se exponen algunas cifras:

1. Dentro del documento, el autor hará referencia a esta entidad mediante su acrónimo. 
A la fecha, según el informe del Observatorio MINED (2018a), solo 1,521 centros escolares tienen conexión a internet, es decir el $34.53 \%$ (de 5,179 instituciones censadas); además, debemos recordar que solo la mitad de la población tiene internet (Yi Min Shum, 2020), y el costo promedio mensual sería no menor a USD 20 (Picardo, Ábrego y Cuchillac, 2020, p. 42)

"La brecha es enorme", señalan Picardo, Ábrego y Cuchillac (2020), agregando que: "surge la pregunta ¿cómo las autoridades van a implementar estrategias de educación a distancia online con estas cifras?" (p. 42).

Acerca de los docentes, una encuesta del 2020 realizada por el MINEDUCYT (citada en Arévalo y Hernández, 2020) afirma que:

[...] un $83 \%$ de los docentes lograron contactar a más de la mitad de sus estudiantes, una vez iniciada la suspensión de clases. Asimismo, se determinó que el personal docente tiene como principal recurso tecnológico el teléfono celular (37.8\%), haciendo uso de los chats $(19.5 \%)$ y redes sociales $(17.6 \%)$ como principal vía de contacto con la comunidad educativa (Arévalo y Hernández, 2020, p. 80).

Y del lado de las familias, dichos investigadores detallan la situación:

No todos los padres de familia del área urbana, y mucho menos rural, poseen teléfonos inteligentes ni cuentan con facilidades de conexión a internet. Durante las primeras semanas, y las que [...] siguieron, el contacto con algunas familias se fue perdiendo paulatinamente, especialmente con aquellas de zonas de mayor precariedad de servicios básicos y de escasos recursos económicos (p. 80).

Ante estas condiciones, Arévalo y Hernández (2020) señalan que "En la práctica, se está desarrollando un proceso de privatización de la educación, [ya que] su gestión tiene que ser mediada por medio de la compra de datos mensuales y equipo tecnológico [...]" (p. 83). Por su parte, Picardo, Ábrego y Cuchillac (2020), a partir de la idea de Iván Ilich, señalan este hecho de una forma radical: "La pandemia de Covid-19 desescolarizó a la sociedad”. Además, "transformó el sistema: La educación dejó de ser pública y gratuita; en efecto, sin internet y sin equipo tecnológico no hay educación" (Picardo, Ábrego y Cuchillac, 2020, p. 40).

Este es el contexto en el que se está desarrollando la educación en El Salvador durante la pandemia: en una sociedad desescolarizada (y la educación se desescolarizó en todo el mundo) y en un sistema prácticamente privado; pues para continuar con la educación se debe pagar por equipo e internet. Ante esto, Arévalo y Hernández (2020) advierten que "la deserción escolar será la consecuencia más palpable de la pandemia al interior del sistema educativo" (p. 73).

Publicado al final del año escolar, el estudio de Picardo, Ábrego y Cuchillac (2020) intenta responder a preguntas como: “ ¿Hubo conti- 
nuidad pedagógica?, ¿qué tan eficiente fue?, ¿se garantizó la calidad necesaria con los sistemas alternativos y online?" [...] "Estos son algunos de los problemas [...] que se están discutiendo entre docentes, padres de familia $y$ estudiantes, y que obviamente demandan una profundización” (p. 41). Arévalo y Hernández (2020) complementan señalando que, "se deben desarrollar procesos de evaluación cualitativos para determinar el impacto educativo en el aprendizaje de los estudiantes" (pp. 73-74).

El estudio de Picardo, Ábrego y Cuchillac resume las acciones tomadas por el MINEDUCYT de este modo:

El Salvador, el 11 de marzo de 2020, suspendió las clases presenciales para todo el sistema educativo público y privado a todo nivel. A partir de ese momento, el MINEDUCYT desarrolló varias estrategias para la continuidad escolar [,] en tres fases:

- Fase 1 (16 de marzo a 3 de abril): se entregaron guías de trabajo elaboradas por los docentes, para todas las asignaturas, a estudiantes, padres y encargados.

- Fase 2 (14 de abril a 15 de mayo): "el Ministerio diseñó guías y materiales didácticos para cada una de las asignaturas, que [...] fueron publicados en sitios oficiales y medios digitales $[y][\ldots]$ entregados de forma impresa a estudiantes con difícil acceso".
- Fase 3 (desde el 25 de mayo hasta el final del año escolar): se siguieron publicando guías de estudio en el sitio del MINEDUCYT y se comenzó a transmitir, como un apoyo a estas, la franja educativa "Aprendamos en casa", a través del Canal 10, la televisión educativa, de carácter estatal (p. 34)².

Como un resultado de su estudio, los investigadores exponen que "Al evaluar la eficiencia del aprendizaje online por materia, en Educación Básica y Media, [...] las materias Lenguaje y Literatura y Ciencias Sociales son en las que se aprende más online” (p. 61).

Asimismo,

Se evaluaron las estrategias diseñadas por el MINEDUCYT para atender el sistema educativo durante la pandemia. [...] los videos de YouTube y libros de texto son las herramientas mejor valoradas; mientras que la franja educativa televisiva y las guías tienen opiniones divididas sobre su efectividad. [...] Al comparar las estrategias del MINED por los sectores educativos público y privado, se observa una gran diferencia; en efecto, [al] sector privado la franja educativa no le ayudó (73.6\%) mientras que en el sector público fue a la inversa, un $75.9 \%$ sí reconoció la ayuda. Lo mismo sucede con las guías educativas. (p. 68)

Acerca de las guías, los investigadores observaron funcionalidad en algunas. Por ejemplo:

2. Arévalo y Hernández (2020) definen esta fase como "Digitalización de la educación [,] que comprende la formación de docentes para el uso de la plataforma Google Classroom” (p. 78). 
"para la asignatura de Lenguaje en Primer Grado, los niños contaban con una guía que proponía lecturas, actividades complementarias y autoevaluación”. Por otro lado, "Otras guías eran muy limitadas en su propuesta pedagógica" (p. 35).

\section{Medición de los aprendizajes}

Sobre la medición global de los aprendizajes en el año escolar 2020, la Prueba de Aprendizaje y Aptitudes para Egresados de Educación Media (PAES) fue sustituida por la prueba AVANZO, que evaluó aprendizajes del 2019 (Primer año de Bachillerato). Picardo (12 diciembre 2020) analiza la prueba y señala las diversas falencias técnicas en su diseño y en sus resultados, señalando que no funciona como prueba estandarizada. Por este motivo, "esta nueva prueba no permitirá medir la continuidad educativa en el nivel y se perderá la trazabilidad de 23 años de medición" (Picardo, Ábrego y Cuchillac, 2020, p. 39). Además, Candray (3 febrero 2021) reporta que los resultados en bruto de la prueba es información "reservada” del MINEDUCYT, así como otros datos relevantes para comprender el impacto de la pandemia en la educación; tales como "la matrícula final 2019[,] la matrícula inicial y final 2020 y la deserción escolar 2019 y 2020”. El investigador también señala que la administración actual del MINEDUCYT tampoco ha continuado con los boletines estadísticos, ni con los observatorios educativos de dicha institución.

\section{Desarrollo}

La literatura más reciente investiga sobre la calidad de la formación docente para la enseñanza de la lectoescritura inicial (LEI) ${ }^{4}$ en Centroamérica (Andrade, Stone y Vijil, 2020) y en El Salvador (Bodewig, 2020), siendo esta última la investigación local que forma parte de la investigación sobre la región. El presente estudio no analiza la continuidad educativa en el nivel educativo inicial (Parvularia); sin embargo, los estudios mencionados son lo más sólido y lo más reciente de lo que disponemos para conocer sobre la formación docente en lectoescritura, y sus resultados dan una medida rigurosa de la formación docente del país en esa área; y (como puede verse más adelante) son extrapolables a otros niveles educativos, por todo lo cual hacemos un resumen de estos para apoyar nuestro marco teórico.

\section{En Centroamérica}

Andrade, Stone y Vijil (2020) adoptan "como perspectiva que la formación del cuerpo docente es el factor clave de la calidad de la enseñanza de la LEI y, por tanto, es fundamental asegurarse que este se prepare como especialista en esta materia” (p. 20). Esta afirmación del docente como clave de la calidad educativa ha sido hecha, también, desde la investigación de la neurociencia sobre el aprendizaje de la lectura (Dehaene, 2019).

3. Apuntamos que este último dato es una constatación de las grandes diferencias entre la educación pública y la educación privada en el país.

4. Dentro del documento, el autor hará referencia a este término mediante su acrónimo. 
A nivel centroamericano, Andrade, Stone y Vijil (2020) resumen la situación sobre la formación docente de este modo:

Una de las principales limitaciones para el avance educativo en los países de Centroamérica es la insuficiente formación de sus docentes. Por lo general, los programas de desarrollo profesional dejan grandes vacíos respecto al dominio de los contenidos a enseñar, de los conocimientos pedagógicos sobre cómo enseñar y de las habilidades para una mediación docente pertinente. Asimismo, es frecuente que la educación del magisterio se diseñe al margen de los planes del sistema escolar y de la formulación de las políticas educativas (p. 1).

Y en las conclusiones de su estudio, las investigadoras afirman que:

Los hallazgos encontrados en los programas de formación docente de cinco países de Centroamérica coinciden con investigaciones previas (Kyeyune et al., 2011; Louzano y Moriconi 2014; OREALCUNESCO, 2013) y muestran [un panorama preocupante por las numerosas brechas identificadas entre cómo se educa al futuro magisterio y lo que la evidencia sugiere $]^{5}$. El estudio revela que la formación inicial que reciben muchos docentes en formación es insuficiente para que enfrenten los retos del trabajo en el aula, y apliquen metodologías efectivas para la instrucción de la LEI. Los vacíos de la formación que esta investigación identificó, limitan la capacidad del futuro docente de abordar las necesidades particulares de la niñez con la que deberá trabajar (p. 20).

\section{En El Salvador}

Bodewig (2020), en el caso particular de El Salvador, señala que "los hallazgos indican que es una formación inicial con fortalezas notables, pero también con brechas y oportunidades de mejora, tanto en lo prescrito en el programa de formación como en la implementación de lo prescrito" (p. 14).

El estudio plantea tres conclusiones principales: 1. el programa de formación docente inicial "se alinea parcialmente a la evidencia investigativa, [...] Sin embargo, el enfoque del programa conduce a un abordaje de dichos ámbitos más desde una mirada conceptual que practica"; 2. los contenidos del programa son limitados para el propósito que se persigue; y 3. el tiempo y "la calidad de las prácticas de enseñanza de la LEI que se ofrecen a futuros educadores" son muy limitados (p. 12).

Acerca de la calidad de las prácticas docentes, la investigadora plantea este panorama crítico:

una de las implicaciones sobre las cuales este estudio quiere llamar la atención, es que el futuro cuerpo de docentes podría estar recibiendo una formación inicial de nivel universitario para enseñar la LEI en un aula de primaria que no conoce; por-

5. El texto colocado dentro de los corchetes son comentarios colocados por el autor. 
que nunca se enfrenta a situaciones reales de instrucción. Cuando alguna vez lo hace, durante su proceso de formación inicial, no tiene oportunidad de observar y modificar sus propios errores a través de prácticas guiadas, modeladas y supervisadas por especialistas en este ámbito clave para la Educación Básica (Bodewig, 2020, p. 13).

Estas y otras limitaciones, con el tiempo se traducirán en una enseñanza bancaria de la lectura y escritura, y en una baja calidad de los aprendizajes (p. 13). Como lo muestra Bodewig:

Estas presunciones de alguna manera se confirmaron en lo observado por Barillas et al. (2005) y Urías (2013) en el estudio que realizaron con docentes en servicio en escuelas públicas. En dichos estudios se reveló la insuficiencia teórica y las serias limitaciones metodológicas de la enseñanza de la LEI: educadores que enseñan a leer y escribir a través de dictados, lectura en coro, transcripción, planas, o preguntas literales después de la lectura, y ausencia de actividades de escritura autónoma. Como se afirma, enseñan en la forma en que fueron enseñados en la escuela y no en su formación inicial docente (Urías, 2013) (Bodewig, 2020, p. 13).

Ante esta situación, las recomendaciones que formula Bodewig (2020) son:

1. [Realizar una formación docente basada en la evidencia científica]. "Es necesa- rio trabajar a nivel nacional en la puesta en práctica de lo que la evidencia sugiere para la enseñanza exitosa de la LEI. Se recomienda ajustar la organización y contenidos del programa de formación, de tal manera que el grupo de formadores que lo implementan tengan lineamientos metodológicos y fundamentación teórica, basada en la literatura científica sobre la enseñanza de la LEI” (p. 13).

2. AActualizar el programa de formación docente]. Las instituciones formadoras de docentes deben actualizar el programa de formación, "a fin de agregar orientaciones metodológicas y teóricas específicas sobre la enseñanza de LEI, pues actualmente no se incluyen" (p. 14).

3. ["Ampliar el tiempo total] que el programa de formación dedica a que el grupo de futuros docentes aprenda [sobre la instrucción efectiva de la LEI]. Esto implica no solo la ampliación de contenidos conceptuales, sino también de tiempo y espacios de prácticas sistemáticas y basadas en el modelaje de cómo enseñar la LEI ${ }^{7 ”}$ (p. 14).

4. A los diseñadores de los programas de formación, tanto del MINEDUCYT como de las instituciones formadoras: "[...] considerar el cambio de enfoque generalista de docentes de educación básica y hacer esfuerzos de [formación de especialistas en la enseñanza de la lectoescritura en los niveles iniciales]" (Bodewig, 2020, p. 14).

6. El texto colocado dentro de los corchetes son comentarios colocados por el autor. 
Por último, sobre la formación docente, ambos trabajos (Andrade, Stone y Vijil, 2020, y Bodewig, 2020) señalan que sus hallazgos buscan promover el diálogo sobre el tema, y que "El conocimiento acumulado debe ampliarse y profundizarse en estudios del mismo tema, que consideren otras perspectivas y metodologías e involucren a más actores clave del sector educativo" (Andrade, Stone y Vijil, 2020, p. 22).

\section{Enseñanza de la lectoescritura en tercer ciclo de Educación Básica y en Educación Media}

Existen pocas investigaciones sobre la enseñanza de la lectoescritura en estos dos niveles en El Salvador. Para Tercer ciclo, encontramos el trabajo de Guerra, Linares y Viana (2013), que analiza la pertinencia de los métodos de análisis literario propuestos en los libros de texto para promover la competencia comunicativa. Entre los resultados, se afirma que:

[...] ninguno de los cuestionarios que aparecen en los libros de texto les dan la oportunidad a los estudiantes de ampliar su visión de mundo a través de las lecturas [,] pues se limitan a la reproducción mecánica y autómata del texto leído sin observar sus características reflexivas (p. 102).

Se registra, además, que la mayoría de los profesores entrevistados para la investigación manifiestan "trabajar solo con los fragmentos literarios que proporcionan los diferentes textos escolares" (p. 204) (es decir que en esos casos no se lee la obra completa); y que "las actividades de fijación sugeridas son cuestionarios, que deberían [de] fortalecer áreas de escritura, destrezas de comprensión lectora; sin embargo [...] no se aplican estrategias adecuadas para que el alumno desarrolle un análisis en profundidad" (p. 204).

Luego, en Educación Media, Iraheta y López (2014), en su análisis del desarrollo de la asignatura de Lenguaje y Literatura afirman que:

[...] existe evidencia suficiente para sostener que los maestros y las maestras de Educación Media del sector público y algunos del sector privado [...] no ponen en práctica de manera adecuada el enfoque educativo por competencias en su trabajo de aula [...] se logró determinar por medio de la observación de campo y las entrevistas, que las formas de enseñar obedecen a un esquema acentuadamente bancario (p. 72).

Por su parte, Aguilar (2012) analiza el papel de la literatura en el desarrollo del pensamiento crítico en Educación Media, identificando una "ausencia de sustentación teórica en los planes y programas, de la noción de pensamiento crítico" (p. 333). Dicho investigador, con base en un estudio de campo, asegura que "la enseñanza de la literatura en la escuela secundaria [salvadoreña] necesita ser repensada” (p. 329). Además de aportar instrumentos para el estudio del desarrollo del pensamiento crítico por medio de la literatu-

7. Acerca de la ampliación de tiempos de formación, también existe la propuesta de "Iniciar una profesionalización docente con una formación inicial de cinco años" (Hernández, citado en Rosales, 21 enero 2021). 
ra, Aguilar (2012) analiza el canon literario de Educación Media, y muestra cómo ha tenido pocos cambios sustanciales en varias décadas. El investigador afirma que "la enseñanza de la literatura [en El Salvador] se realiza con base en un enfoque historicista, europeizante y masculino" (p. 173ss). Tercer ciclo utiliza un canon literario que también puede ser caracterizado de este modo.

Para concluir con la revisión de la literatura, se puede decir que la enseñanza de la lectoescritura en Educación Básica y Media en El Salvador cuenta con fortalezas, tales como planes de formación docente y currículos basados en el enfoque en competencias. Sin embargo, la aplicación de estos planes y currículos encuentra graves obstáculos, tales como limitaciones en los formadores de docentes, limitaciones teóricas y de práctica curricular; una persistencia en la enseñanza de tipo bancario y, en los casos de Tercer ciclo de Educación Básica y de Educación Media, un enfoque de enseñanza de la literatura rígido y conservador, con grandes limitaciones para promover el desarrollo de competencias y capacidades en los estudiantes.

\section{La enseñanza de la lectoescritura durante la pandemia}

Para iniciar el análisis de la enseñanza de la lectoescritura en El Salvador durante la pandemia de COVID-19, se hace un resumen de la revisión de la literatura, con los datos que se consideran principales. Así, según los estudios:
1. La estrategia de gestión de los procesos educativos ha sido centralizada por el MINEDUCYT (Arévalo y Hernández, 2020; Picardo, Ábrego y Cuchillac, 2020).

2. En el sector público, los procesos educativos realizados en este período pueden caracterizarse como de corte bancario, mediados por el uso de internet (Arévalo y Hernández, 2020, p. 74).

3. El MINEDUCYT "se enfocó en la 'continuidad del desarrollo del currículo', sin matizar con elementos de contexto [de la situación actual] para promover un aprendizaje más significativo en el estudiantado" (Arévalo y Hernández, 2020, p. 81).

4. Se reporta la formación de docentes del sector público para el uso de la plataforma Google Classroom (Arévalo y Hernández, 2020, p. 78).

5. Las materias Lenguaje y Literatura y Ciencias Sociales son en las que se aprendería más online (Picardo, Ábrego y Cuchillac, 2020, p. 61).

6. Se observó funcionalidad en algunas guías de aprendizaje (se muestra el caso de una de Lenguaje y Literatura) y se encontró que otras "eran muy limitadas en su propuesta pedagógica" (Picardo, Ábrego y Cuchillac, 2020, p. 35).

A partir de estos datos, se analizaron tres elementos: la formación docente, los materiales didácticos y las teleclases de Lenguaje y Lite- 
ratura; observando en especial en estos dos últimos: 1. la promoción de competencias y capacidades frente a una educación bancaria; y 2. la contextualización de los aprendizajes frente a la continuidad del currículo prescrito.

\section{a. Formación docente}

Asumimos la formación docente como el factor clave para la calidad educativa (UNESCO, 2017; Andrade, Stone y Vijil, 2020), y desde esta postura nos preguntamos: ¿cómo se está realizando la formación docente, tanto la continua como la inicial, durante pandemia? Durante el 2020, se reporta la capacitación en el uso de la plataforma Google Classroom (Picardo, Ábrego y Cuchillac, 2020); y en el presente año, se han inaugurado laboratorios de grabación, multimedia y capacitación para educación virtual (MINEDUCYT, 4 febrero 2021). Queda pendiente la tarea de dar seguimiento a estas y otras acciones de formación docente.

\section{b. Programas de estudio}

Existe una diferencia entre los programas de estudio y algunos materiales didácticos utilizados en 2020 y los de 2021. En 2020, el MINEDUCYT se apoyó principalmente en las guías de aprendizaje diseñadas por especialistas de la institución ${ }^{8}$; mientras que en 2021, se incorporan como apoyo libros de texto, elaborados en el marco del proyecto FOMILENIO II (FOMILENIO II, 22 julio 2020).
Estos libros de texto forman parte de la implementación de nuevos planes de estudio (MINEDUCYT, 2021a). A la nueva colección de libros para Lenguaje y Literatura se la ha llamado ES Lengua. Hasta la fecha, se han subido al sitio del MINEDUCYT los programas de estudio y los libros de texto de Primer grado y de Tercer ciclo de Educación Básica $\left(7^{\circ}\right.$, $8^{\circ}$ y $9^{\circ}$ grados); y se señala al final de estos que son material en validación (MINEDUCYT, 26 enero 2021$)^{9}$.

\section{c. Competencias}

Tanto el programa anterior (MINED, 2008) como el actual (MINEDUCYT, 2021a), declaran en su presentación estar basados en el enfoque por competencias, y tener el enfoque comunicativo como orientación específica de la asignatura. Una diferencia es que el programa nuevo enuncia como su meta concreta la competencia comunicativa ${ }^{10}$.

Hay que apuntar que, sobre la enseñanza con enfoque en competencias en el país, como se muestra en apartados anteriores, los estudios plantean que - en general - esta no se ha hecho efectiva, siendo el factor principal una formación docente que no se ha habilitado para ello (Iraheta y López, 2014; Andrade, Stone y Vijil, 2020; Bodewig, 2020). Hay que señalar que, junto con la necesaria renovación de los programas de estudio, debe llevarse a cabo una formación docente que se habilite para utilizar dichos programas efectivamente.

8. Las guías de aprendizaje de 2020 se pueden consultar en: https://sites.google.com/clases.edu.sv/educacionvirtual/ inicio?authuser $=0$. Se encuentran disponibles las guías de las fases 1 y 3 de la continuidad educativa. Consultado el 15 febrero 2021.

9. Esta información se verificó por última vez el 15 febrero 2021.

10. La validez del enfoque comunicativo para Lenguaje y Literatura ha sido discutida por Aguilar (2016). 
Hasta ahora, dicha acción conjunta no se ha realizado, pero es indispensable para garantizar la calidad educativa.

Ahora, volviendo a los programas en sí, ambos procuran básicamente las mismas competencias: comprensión oral, expresión oral, comprensión lectora y expresión escrita. El programa nuevo ha eliminado la competencia que el anterior denominaba comunicación literaria (MINED, 2008, p. 9). Esto se evalúa como un acierto, pues sus elementos están comprendidos en las competencias ya mencionadas, que son básicas para el aprendizaje de la lectoescritura.

\section{d. Lineamientos metodológicos}

En el aspecto de los lineamientos metodológicos, los programas nuevos proponen una secuencia didáctica para el desarrollo de las clases (MINEDUCYT, 2021a, pp. 10-11). Dicha secuencia sigue un esquema $\mathrm{ACC}^{11}$ : anticipación (activación de saberes previos), construcción (realización de actividades que ayudan al desarrollo de aprendizajes nuevos) y consolidación (aplicación de "los conocimientos construidos en la resolución de actividades genuinas (contextualizadas) con demanda cognitiva"). Esta secuencia, en tanto sea un procedimiento que los docentes dominen, puede brindar aportes.

Sin embargo, estos mismos lineamientos metodológicos nuevos contienen un punto que debe ser analizado con detenimiento.
El programa plantea en dicho apartado: "El aprendizaje de la lengua con enfoque comunicativo requiere metodologías innovadoras y participativas, que sitúen a cada estudiante como protagonista del proceso de aprendizaje, orientado por el o la docente" (p. 9). Hasta este punto, el planteamiento es positivo y acorde a las realidades actuales. Continúa: "[El aprendizaje de la lengua con enfoque comunicativo] Requiere una gestión de aula enmarcada en una escuela activa y un modelo de enseñanza explícita [...]” (p. 9). El modelo de enseñanza explícita, según explica el programa, busca claridad en el desarrollo de los procesos de enseñanza aprendizaje:

La instrucción o modelo de enseñanza explícita se caracteriza por una serie de soportes y orientaciones, por los cuales el estudiando es guiado en el proceso de aprendizaje. Esta constituye declaraciones claras sobre el propósito y las razones para aprender la nueva habilidad o desarrollar una competencia; explicaciones precisas para resolver una tarea simple o compleja, demostraciones del objetivo de instrucción y prácticas con retroalimentación hasta que se haya alcanzado el dominio independiente (p. 10).

Hasta aquí los planteamientos del programa son pedagógicamente funcionales. La cuestión surge cuando, al continuar, se afirma que:

[El aprendizaje de la lengua con enfoque comunicativo] Requiere una gestión de

11. Dentro del documento, el autor hará referencia a este término mediante su acrónimo. 
aula enmarcada en una escuela activa y un modelo de enseñanza explícita [que libere a la y al docente de las interpretaciones curriculares y le brinde indicaciones claras sobre qué, cómo, con qué y para qué enseñar $]^{12}$, así como una secuencia didáctica que permita vincular los conocimientos y experiencias previas con los nuevos aprendizajes (p. 9).

Haciendo nuestra interpretación de este planteamiento, lo que nosotros comprendemos es que dicha liberación del docente de las interpretaciones curriculares se traduciría en dirigir en todo lo posible las acciones de este en su práctica pedagógica... Lo cual no es una liberación. Por otro lado, si a lo que se refiere el planteamiento es a liberar a los docentes de las interpretaciones curriculares arbitrarias, ocurrentes, no pedagógicamente pertinentes, las acciones necesarias para ello deben realizarse, como un fundamento funcional, en los procesos de formación docente.

Se sugiere revisar en los programas de estudio y en las concepciones del MINEDUCYT, planteamientos como el observado, pues su puesta en práctica limita la autonomía docente, lo desprofesionaliza al imponerle una racionalidad técnica (Stenhouse); y sería una confirmación del carácter centralizador de la gestión educativa y restrictiva de la práctica docente, en este período que otros estudios han señalado (Picardo, Ábrego y Cuchillac, 2020; Arévalo y Hernández, 2020).

\section{e. Contenidos}

En el aspecto de los contenidos, el programa nuevo muestra una amplitud mayor que el anterior (ver figura 1).

El programa de $7^{\circ}$ grado de 2021 aborda los diversos géneros literarios, en lugar de enfocarse solo en los géneros narrativos. Al mismo tiempo realiza algunas adecuaciones al desarrollo cognitivo e intereses de los estudiantes como, por ejemplo, retirar el estudio de la novela de caballería y la novela picaresca.

Además, al revisar el contenido de las unidades, se pueden encontrar diversos cambios positivos:

\section{- Repositorios y bibliotecas virtuales de acce-} so abierto: uso y estructura. (Unidad 1).

- Textos digitales. El blog literario: estructura y función. (Unidad 2).

- La recepción de textos poéticos: Poemas de César Vallejo y Claribel Alegria. (sic). (Unidad 4).

Con los primeros dos contenidos, se está dando una actualización de los aprendizajes deseados; y con el tercero, se hace un avance para cambiar el carácter eurocéntrico y masculino del canon literario en la escuela salvadoreña (Aguilar, 2012 y 2013).

Como un punto de mejora se observa el orden de los contenidos. Por ejemplo, el contenido "La producción de textos de interacción,

12. El texto colocado dentro de los corchetes son comentarios colocados por el autor. 


\begin{tabular}{|c|c|c|}
\hline $\begin{array}{c}\text { Número de } \\
\text { Unidad }\end{array}$ & Programa 2008 & Programa 2021 \\
\hline 1 & Narrativa: El cuento maravilloso & El cuento maravilloso \\
\hline 2 & Narrativa: El cuento de terror & El mundo de la novela \\
\hline 3 & Narrativa: El cuento policial & Conozcamos el teatro \\
\hline 4 & Narrativa: El cuento realista & La poesía \\
\hline 5 & Narrativa: El cuento de ciencia & El cuento de terror \\
\hline 6 & Narrativa: El cuento surrealista & El mundo de la ciencia ficción \\
\hline 7 & $\begin{array}{r}\text { Narrativa: La novela de caballería } \\
\text { y la novela picaresca }\end{array}$ & Información en movimiento \\
\hline 8 & Narrativa: La novela romántica y & A la luz de las palabras \\
\hline
\end{tabular}

Figura 1. Contenido de programas de estudio de Lenguaje y Literatura de $7^{\circ}$ grado.

Fuente: Elaborado a partir de los datos del MINED (2008) y MINEDUCYT (2021a).

los mensajes en redes sociales: los chats y el correo electrónico, con el uso del lenguaje icónico y verbal" se encuentra hasta $9^{\circ}$ grado, cuando los usos del chat y del correo electrónico - que son formas básicas de comunicación a través de las TIC - deben enseñarse más temprano. Efectivamente, cuando el consumo y uso de redes sociales se está dando más temprano.

\section{f. Libros de texto}

Los libros de texto para la educación pública eran una necesidad acuciante, pues la última colección de estos (Colección Cipotas y Cipotes), llevaba varios años sin ser reimpresa.
En el libro de ES Lengua de $7^{\circ}$ grado (MINEDUCYT, 2021b), se observan las siguientes características:

- Posee un diseño atractivo.

- Los aprendizajes esperados se enuncian al inicio de cada unidad.

- Desarrolla secuencias didácticas según el esquema ACC, planteado en el Programa de estudios (MINEDUCYT, 2021a, pp. 10-11).

- Plantea la elaboración de un producto semanal, así como criterios para su evaluación.

- Posee recuadros con información adicional e hipervínculos. 


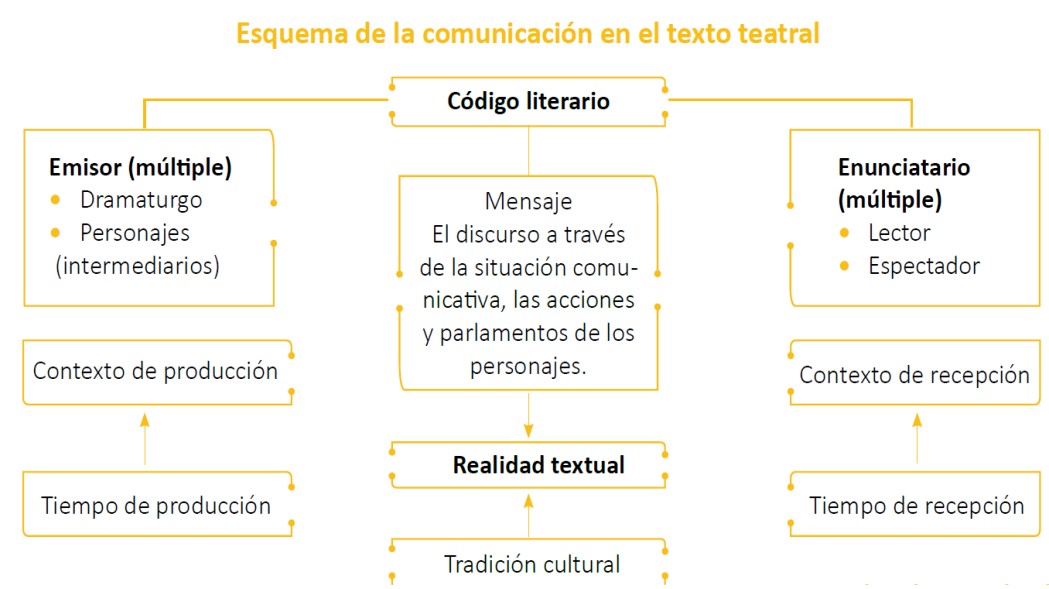

Figura 2. Esquema de la comunicación en el texto teatral.

Fuente: Libro de texto de $9^{\circ}$ grado, Colección ES Lengua (MINEDUCYT (2021c, p. 100).

Hacemos dos observaciones de puntos de mejora. La primera es que estos libros contienen un exceso de teorización (ver figura 2).

Surge la pregunta: ¿realmente son estos los aprendizajes que se deben desarrollar sobre, por ejemplo, el teatro, en $9^{\circ}$ grado? De este modo, los libros de texto, así como los programas de estudio, al igual que su versión anterior (de 2008), estarían buscando “enseñar” teoría de un nivel bastante especializado saberes que no serían pertinentes para desarrollar las competencias de lectoescritura básicas, ni las capacidades de participación en la cultura que estos contenidos tienen el potencial de propiciar, si se los aborda de modo significativo.

Y la segunda observación es que, las indicaciones para los estudiantes, al estar redactadas en primera persona, son demasiado directivas. Se muestra como ejemplo algunas indicaciones:

- "Actividad individual. Respondo". (Al constar la actividad de preguntas e indica- ciones, seguidas de líneas en blanco, es evidente para el estudiante que se le pide una respuesta escrita).

- "Escribimos qué entendemos por comunicación".

- "Leemos el esquema de la comunicación y socializamos una explicación de cada uno de sus elementos" (MINEDUCYT, 2021b, p. 10).

\section{g. Guías de aprendizaje}

Una revisión de los materiales didácticos del MINEDUCYT para la continuidad educativa durante la pandemia, permite comprobar que en el 2021 se están utilizando las mismas guías de aprendizaje del 2020 (Comparar MINEDUCYT, s.f.a con MINEDUCYT, s.f.b). En las orientaciones generales para docentes, el Ministerio de Educación plantea que:

Las guías de la fase 1 de 2021 retoman indicadores de logro y contenidos del programa de estudios vigente [que sería el de 
2008]. A partir de la octava semana, se iniciará el desarrollo de actividades a través del libro de texto de la estrategia ESLengua, el cual está organizado por semanas didácticas. En este contexto, las guías presentan actividades de cierta complejidad para que el alumnado logre fijar los aprendizajes, y tenga las competencias para iniciar el desarrollo de sus aprendizajes con la estrategia ES Lengua; cuyo propósito es que el estudiantado alcance las competencias transversales y de ciclo, a través de la contextualización del currículo y su pertinencia ante los cambios de la comunicación digital (MINEDUCYT, 2021d).

Sobre las guías de 2020, como se ha mencionado anteriormente, se observó funcionalidad en algunas (por ejemplo: "para la asignatura de Lenguaje en Primer grado, los niños contaban con una guía que proponía lecturas, actividades complementarias y autoevaluación"; y, por otro lado, "Otras guías eran muy limitadas en su propuesta pedagógica" (Picardo, Ábrego y Cuchillac, 2020, p. 35).

Dichas guías, al igual que los programas de estudio en los que están basados (MINED, 2008) contienen una teorización excesiva. Por ejemplo, los contenidos de la primera guía de $7^{\circ}$ grado son:

- El texto literario. Características.

- La comunicación literaria. Función poética de la lengua. Los elementos de la comunicación literaria (MINEDUCYT, 2021e).
Habría más posibilidades de involucramiento, emocional y cognitivo de los estudiantes, al desarrollar temas como "El mundo de la literatura” (en lugar de El texto literario) y “Cómo se escribe la literatura?” o "La magia de la literatura. ¿Cómo escribir un cuento?”. Además, en Educación Media, las guías de 2020 siguieron el canon literario escolar del programa de estudios de 2008, que Aguilar (2012 y 2013) ha determinado como infuncional en diversos aspectos; caracterizándolo - de manera general - como basado en un enfoque historicista, europeizante y masculino (Aguilar, 2013, p. 173ss). Estas características también pueden hallarse en el canon de Tercer ciclo de ese programa.

\section{h. Teleclases}

El Ministerio de Educación afirma que las teleclases son "el mismo material de las guías explicado por los mismos especialistas, utilizando recursos como animaciones, títeres, actividades lúdicas, entre otros; garantizando el aprendizaje desde el hogar" (MINEDUCYT, 24 mayo 2020).

En la asignatura de Lenguaje y Literatura, las teleclases brindan información complementaria o interesante sobre los contenidos tratados; sin embargo, en general, tienen como puntos de mejora los mismos de las guías: uno notable es el exceso de teorización. El autor de este artículo recogió la opinión de una exalumna que el año pasado cursaba Segundo año de Bachillerato: "en algunos momentos, el lenguaje que los maestros usan (no 
solo en esta materia [Lenguaje y Literatura], si no, [sic] en todas) es muy técnico; y por momentos es difícil llevar la continuidad de la idea" (comunicación personal, 10 de junio de 2020).

Por último, al repetir en las teleclases de Lenguaje y Literatura el contenido de las guías de aprendizaje (y en muchas ocasiones, literalmente, palabra por palabra), la capacidad pedagógica de estas se ve limitada, y se identifica una enseñanza transmisionista, bancaria ${ }^{13}$.

\section{Conclusiones}

Al hacer un análisis de la enseñanza de la lectoescritura en El Salvador durante la pandemia de COVID-19, en los niveles de Educación Básica y Educación Media, se concluye que:

- La enseñanza de la lectoescritura, en ambos niveles, posee como limitación principal la formación docente; que actualmente no habilita de manera funcional para este fin. Es necesario emprender acciones estructuradas a corto, mediano y largo plazo para mejorar dicha formación docente, tanto inicial como continua. Entre estas acciones se encuentran: la creación y la puesta en práctica de políticas educativas; el mejoramiento de los planes de las carreras de profesorado; una formación y una práctica docentes basadas en la evidencia; y la práctica observada y con retroalimentación, en entornos reales, como parte de la formación docente. Los estudios de Andra- de, Stone y Vijil (2020) y Bodewig (2020) buscan promover el diálogo sobre el tema y proporcionan una bibliografía actualizada y nutrida sobre el mismo.

- Las acciones adoptadas para la continuidad y mejora de la enseñanza de la lectoescritura, como son el diseño de guías de aprendizaje, la implementación de un nuevo programa de estudios y de nuevos libros de texto, presentan puntos de funcionalidad, y también puntos de mejora que es necesario atender. Entre los puntos funcionales se encuentran la propuesta de una secuencia didáctica para las clases, la actualización de los aprendizajes (por ejemplo con la comunicación a través de las TIC) y modificaciones en el canon literario escolar (durante décadas historicista, eurocéntrico y masculino). Entre los puntos de mejora se identifica la persistencia de un tratamiento demasiado teórico de los contenidos. Se apunta que las mejoras señaladas en el programa de estudios aún deben profundizarse en otras investigaciones, pues aquí han sido revisadas de un modo general.

- Durante la pandemia, al analizar los programas de estudio, las guías de aprendizaje y las teleclases se concluye que, en la asignatura de Lenguaje y Literatura, en el sector público, más que el desarrollo de competencias de lectura y escritura, se ha dado - hasta este punto, y de acuerdo con la observación de Arévalo y Hernández (2020) - una educa-

13. Las teleclases de Lenguaje y Literatura de 2021 se pueden ver en el canal de Youtube: "Aprendamos en casa El Salvador", en el siguiente enlace: https://www.youtube.com/playlist?list=PLXlEcrcdXySrFAuTYQiDnfBxNQkUEY_9v 
ción bancaria, transmisionista; mediada por internet. En este sentido, las guías de estudio y las teleclases tendrían una gran influencia, probablemente decisiva, tanto en los aprendizajes de los estudiantes al interactuar con estos materiales, como en el trabajo de los docentes con los estudiantes. Esto último, en la medida en que los docentes se ciñan a las indicaciones de los materiales, con lo cual se perfilaría una enseñanza más bancaria (en tanto más se ajuste a las guías) o más significativa (si así se diseñan las actividades de aprendizaje). Este hecho corrobora la afirmación de Arévalo y Hernández (2020); y Picardo, Ábrego y Cuchillac (2020), de que el Ministerio de Educación ha tenido una actuación centralista durante la pandemia. Es necesario generar unas directrices generales y un acompañamiento constante al docente durante esta contingencia, pero la contextualización efectiva de los aprendizajes, basada en el conocimiento de los estudiantes y de su entorno, solo puede ser llevada a cabo por el docente; para lo cual requiere autonomía y, como ya hemos señalado en el artículo, una formación que lo habilite para una enseñanza de calidad: Una enseñanza orientada a lograr aprendizajes significativos; además de que, una formación así, también hace posible la autonomía.

También es necesario observar cómo la educación basada en competencias es un paradigma que aún no se comprende ni se aplica en la escuela salvadoreña. Una prueba de esto es que se siguen "enseñando contenidos", y en ese contexto, la competencia se ve como un proceso de "simple reproducción del conocimiento o empleo de herramientas" (Tobón, 2014 , p. 26); cuando en realidad, las competencias "implican la creatividad, la flexibilidad y el afrontamiento de situaciones diversas y de incertidumbre" (Tobón, 2014, p. 26).

Una experiencia exitosa de innovación educativa en El Salvador es la de Giménez (2014), cuyo equipo implementó la Metodología de Aprendizaje por Proyectos con docentes salvadoreños, quienes se apropiaron de esta en sus diversos ámbitos educativos. Estas experiencias, registradas en el trabajo de dicha autora muestran casos de proyectos integrados, que pusieron en juego los saberes de diversas asignaturas para la construcción de conocimientos y el logro de objetivos grupales. Esta es una experiencia local que demuestra cómo el logro de la calidad educativa es posible a través de procesos de innovación estructurados y participativos.

Por último, las acciones para mejorar la enseñanza de la lectoescritura durante la pandemia deben contemplar, también, los medios para superar la considerable brecha digital educativa del país. 


\section{Referencias}

Aguilar-Ciciliano, M. (2012). El papel de la literatura en el desarrollo del pensamiento crítico de las y los estudiantes de educación media: el caso salvadoreño [Tesis de doctorado]. Universidad de Costa Rica.

Aguilar-Ciciliano, M. (2013). Canon literario escolar y enseñanza de la literatura en la educación media: Un análisis crítico de los programas de enseñanza secundaria en El Salvador. Educare, 17(3), 173-198. https://doi.org/10.15359/ree.17-3.9

Aguilar-Ciciliano, M. (2016). El concepto de la lectura literaria en la pedagogía actual de la literatura. La Universidad, 31, 63-85. http://revistas.ues.edu.sv/index.php/launiversidad/ article/view/765

Andrade-Calderón, P.; Stone, R. y Vijil, J. (2020). ¿Cómo se está formando al cuerpo docente centroamericano para enseñar la lectoescritura inicial? Aportes de una investigación regional a partir de cinco estudios de caso. Revista Actualidades Investigativas en Educación, 20(2), 1-33. Doi. 10.15517/aie.v20i2.41588

Arévalo, A. y Hernández-Pereira, R. (2020). ¿Educación en cuarentena? gestionando procesos educativos en medio de la pandemia de COVID-19 en El Salvador. Revista Interdisciplinaria de Estudios Latinoamericanos, 4(3), 73-86.

Blanchard-Giménez, M. (Coord.). (2014). Transformando la sociedad desde las aulas. Metodología de Aprendizaje por Proyectos para la innovación educativa en El Salvador. Madrid: Narcea.

Bodewig, C. (2020). Formación Inicial Docente sobre Lectoescritura Inicial en la Licenciatura en educación básica para Primer y Segundo ciclo que ofrece la Universidad Centroamericana José Simeón Cañas, El Salvador. Revista Actualidades Investigativas en Educación, 20(2), 1-16. Doi. 10.15517/aie.v20i2.41593

Candray, J. (3 febrero 2020). Las “políticas de uso y privacidad” de la información pública han cambiado. Disruptiva. https://www.disruptiva.media/las-politicas-de-uso-y-privacidadde-la-informacion-publica-han-cambiado/

Dehaene, S. (2019). Aprender a leer: De las ciencias cognitivas al aula. Siglo XXI Editores. (Ebook).

FOMILENIO II (22 julio 2020). FOMILENIO II invierte \$14.6 millones para formación de docentes y nuevos planes de estudio. https://www.fomilenioii.gob.sv/fomilenio-ii-invierte-14-6-millonespara-formacion-de-docentes-y-nuevos-planes-de-estudio.html\#: :text=FOMILENIO\%20II\%20 ha\%20invertido\%20\%2414.6,de\%20aprendizajes\%20y\%20evaluaci\%C3\%B3n\%20curricular. 
Guerra-Heredia, G. I.; Linares, T. E y Viana-Guzmán, K. I. (2013). Manual didáctico de análisis literario para $7^{\circ}, 8^{\circ}, 9^{\circ}$ grados en Lenguaje y Literatura de Educación Básica [Tesis de Licenciatura]. Universidad de El Salvador. http://ri.ues.edu.sv/id/eprint/13202

Hernández-Pereira, R. En: Rosales, D. (21 enero 2021). Esperar 15 años por una plaza. La Prensa Gráfica. https://7s.laprensagrafica.com/esperar-15-anos-por-una-plaza/

Iraheta, I. M. y López, I. E. (2014). Análisis del enfoque educativo por competencias aplicado en la enseñanza de Lenguaje y Literatura en el Programa de Estudio de Educación Media en El Salvador. Universidad de El Salvador, Facultad Multidisciplinaria de Occidente [Tesis de Licenciatura]. http://ri.ues.edu.sv/id/eprint/13077

MINEDUCYT (s.f.a). Educación en línea. (Sitio de Google Sites con los materiales didácticos de la continuidad educativa en 2020). https://sites.google.com/clases.edu.sv/educacionvirtual/ inicio?authuser $=0$

MINEDUCYT (s.f.b). (Sin título). (Micrositio del MINEDUCYT para la continuidad educativa en 2021). https://www.mined.gob.sv/continuidadeducativa/

MINED (2008). Programas de estudio. Lenguaje y Literatura. Tercer Ciclo de Educación Básica. https://www.mined.gob.sv/descargas/category/869-programas-de-estudio-para-tercerciclo-de-educacion-basica.html

MINEDUCYT (24 mayo 2020). Ministra de Educación verifica producción de franja educativa «Aprendamos en Casa». https://www.mined.gob.sv/noticias/item/1015529-ministra-aprendamos-en-casa.html

MINEDUCYT (2021a). Programas de estudio. Lenguaje y Literatura. Tercer Ciclo de Educación Básica. (PDF). https://www.mined.gob.sv/eslengua/Programa\%20Lenguaje\%20Tercer\%20 ciclo\%202021.pdf

MINEDUCYT (2021b). 7. Lenguaje y Literatura. Libro de texto. Colección ES Lengua. (PDF). https://www.mined.gob.sv/eslengua/Libro\%20de\%20texto\%207.\%C2\%B0.pdf

MINEDUCYT (2021c). 9. Lenguaje y Literatura. Libro de texto. Colección ES Lengua. (PDF). https://www.mined.gob.sv/eslengua/Libro\%20de\%20texto\%209.\%C2\%B0.pdf

MINEDUCYT (2021d). Orientaciones generales para docentes. Tercer ciclo. https://www.mined. gob.sv/materiales2021/f1/semana1/7grado/Lenguaje\%20y\%20Literatura/Guia_autoaprendizaje_estudiente_7mo_grado_Lenguaje_f1_s1.pdf 
MINEDUCYT (2021e). Lenguaje y Literatura. Guía de continuidad educativa. Estudiantes $7^{\circ}$ grado. Fase 1, semana 1. https://www.mined.gob.sv/materiales2021/f1/semana1/7grado/Lenguaje\%20y\%20Literatura/Guia_autoaprendizaje_estudiente_7mo_grado_Lenguaje_f1_s1.pdf

MINEDUCYT (26 enero 2021). ESLengua. https://www.mined.gob.sv/materiales-educativos/ item/1016038-eslengua.html

MINEDUCYT (4 febrero 2021). Viceministro de Educación inauguró los laboratorios de Multimedia para Educación Virtual en la UTEC. https://www.mined.gob.sv/noticias/item/1016051viceministro-de-educacion-inauguro-los-laboratorios-de-multimedia-para-educacionvirtual-en-la-utec.html

Picardo, O. (12 diciembre 2020). AVANZO: Buen espectáculo, mala prueba... Disruptiva. https://www.disruptiva.media/avanzo-buen-espectaculo-mala-prueba/

Picardo, O.; Ábrego, A. M. y Cuchillac, V. (2020). Educación y COVID-19: Estudio de factores asociados con el rendimiento académico online en tiempos de pandemia (caso El Salvador). Universidad Francisco Gavidia. http://hdl.handle.net/11592/9645

Tobón, S. (2014). Proyectos formativos. Teoría y metodología. Ciudad de México: Pearson.

UNESCO. (2017). Desglosar el Objetivo de Desarrollo Sostenible 4. Educación 2030. Guía. https://unesdoc.unesco.org/ark:/48223/pf0000246300_spa/PDF/246300spa.pdf.multi 